\title{
Partial volume correction for approximating crack opening displacements in CFRP material obtained from micro-focus X-ray CT scans \\ D.J. Bull ${ }^{1 *}$, I. Sinclair ${ }^{1}$, S.M. Spearing ${ }^{1}$ \\ ${ }^{1}$ Materials Research Group, Faculty of Engineering and the Environment, University of Southampton, Southampton, United Kingdom \\ * Corresponding author (daniel.bull@soton.ac.uk)
}

Keywords: A. Carbon fibre, B. Impact behaviour, C. Crack; D. Non-destructive testing; X-ray computed tomography

\section{Abstract}

This paper presents a partial volume correction technique that applies a measurement weighting based on grey scale intensity values, allowing crack opening displacements (CODs) to be better estimated in micro-focus computed tomography ( $\mu \mathrm{CT})$ scans. These were tested on 3D data obtained from two separate $\mu \mathrm{CT}$ scanners on particle toughened and non-particle toughened carbon fibre material subjected to low velocity impact. Direct comparisons of COD estimations were made with higher resolution measurements obtained using synchrotron radiation computed tomography (SRCT) scans taken at the European Synchrotron Radiation Facility (ESRF). In this study, partial volume correction is reported to improve the accuracy of these measurements to within $20 \%$ of SRCT measurements, whereas measurements based on counting interconnected voxels representing a detectable crack are reported to consistently overestimate crack openings by up to $500 \%$. Scatter in estimations was dependent on material type, noise, and artefacts associated with $\mu C T$ volumes. 


\section{Introduction}

Industrial CT scanners have made it possible to routinely extract 3D damage features in structural materials such as carbon fibre composites [1]. Common operating voxel resolutions of the order of 5 microns have been used in previous studies on impacted composites [2-7]; the significant limiting factor affecting resolution is the X-ray focal spot size and specimen size [8]. Whilst this resolution is useful for identifying the components of composite damage, quantification of key features, such as crack opening displacements (CODs) within impacted carbon fibre materials requires a higher fidelity. Informed use of the partial volume effect provides a means so that sub-resolution features may still be detected and quantified [2]. Crack opening and crack shear displacements are important parameters in micromechanical modelling of composite damage and failure. Accurate experimental measurement of these parameters in three-dimensions (3D) is enabled by computed tomography, and allows the validation and calibration of models [9] and to calculate effective stress intensities at the crack tip [10].

The partial volume effect occurs when two or more phases with differing density are represented within a single voxel leading to an effective averaging of attenuation coefficients; this is typically critical if the object or region's dimensions are at the voxel resolution or less (assuming other forms of unsharpness are undersampled by the voxel dimensions) [11-13]. Depending on the contrast difference between the two phases, this has an influence on the smallest detectable feature. For the detectability of cracks in composites it has been reported that openings down to $20 \%$ of the voxel resolution in CT scans can be detected, and by using contrast enhancement agents, this may be further reduced to $5 \%$. Contrast agents require all cracks to be interconnected up to the surface of 
the material to allow full penetration of the dye [2]. For internal damage, such as that sustained in impact loading, this is often not the case. Furthermore, in cases for which in situ load-stepped CT experiments are combined with digital volume correlation (DVC), the detectability of cracks can be greatly improved by quantifying the mechanical effects of cracking in addition to direct physical imaging $[14,15]$. However, these rely on comparisons between multiple scans rather than a single volume.

The partial volume averaging effect may lead to inaccuracies in the estimation of object sizes due to the dependence on the feature's location on the image grid. Figure $1(a$ and $b)$ shows schematically how this affects crack opening measurements. Since a crack that partially fills a voxel may be treated as occupying a full voxel when interconnected measurements are taken, overestimations in measurements occur [16-18]. This phenomenon is particularly noticeable at phase boundaries that fall within a voxel, leading to intermediate voxel intensities dependant on the percentage of "fill" between both phases $[16,19,20]$.

Where the crack openings approach the limit of detectability, in reported cases down to $20 \%$ of the voxel resolution, measurements of counting interconnected regions will overestimate the crack opening by up to ten times. If the smallest detectable crack were to partially fill two neighbouring voxels, measurements would include the crack as fully occupying both voxels leading to a large overestimation. Scanning at higher voxel resolutions does reduce these errors [16], however the trade-off between resolution and field of view [8], and the high barriers to entry to equipment capable of higher resolution such as synchrotron radiation computed tomography (SRCT) often limits this option. 
A partial volume correction algorithm has been used in previous studies to estimate crack openings on Al-Li fatigue cracks in work by Ignatiev et al. [21] and Guvenilir et al [22, 23]. This work utilised attenuation coefficients to calculate an estimate for the measured fraction of crack opening. Work similar to this by Heckel et al. [19] utilised linear interpolation based on intensity and applied a weighting on the voxel volume at feature boundaries; this has been reported to increase accuracy and repeatability in volume measurements of liver metastases and lymph nodes obtained in CT scans.

The work presented in the present paper uses the same techniques by mapping grey scale intensities to a linear relationship between the material and crack levels to allow an adjusted length to be calculated for that voxel representing a crack opening. To the author's knowledge, this is the first study applying this technique on cracks in carbon fibre composite materials and unlike previous work on this topic, this paper calibrates partial volume correction estimates to higher voxel resolution SRCT scans enabling this technique to be tested more rigorously.

\section{Methodology}

\subsection{Specimens and Impact Testing}

Two carbon fibre reinforced epoxy materials were tested; one with a particle toughened and the other with a non-particle toughened (untoughened) matrix system (exact chemical formulation is proprietary). This allowed different damage morphologies to be examined for crack opening displacement (COD) analysis. The specimens measured $80 \times 80 \times 1 \mathrm{~mm}$ with an eight ply [+45/0/-45/90 $]_{s}$ stacking sequence. Damage was introduced via impact using a drop tower with a $4.9 \mathrm{~kg}$ mass with a $16 \mathrm{~mm}$ diameter hemispherical tup. The specimens 
were supported on a base plate consisting of a $60 \mathrm{~mm}$ diameter hole following a similar standard to that used in [24]. Specimens were impacted at $0.6 \mathrm{~J}$ and $1.3 \mathrm{~J}$ for particle toughened and untoughened systems respectively; this was to create a similar projected damage area in each, as measured by C-scan. These were subsequently cut to form 'matchsticks'; i.e. specimens measuring $80 \times 4.5 \times 1 \mathrm{~mm}$, sized to fit within the field of view of the CT scan at the highest resolutions and to minimise noise and artefacts. To reduce variations in X-ray path length as the sample was rotated during the scan, two specimens were stacked together to form a stack measuring $80 \times 4.5 \times 2 \mathrm{~mm}$.

\subsection{D X-Ray Tomography}

$\mu C T$ scans on the 'matchstick' samples were carried out at the University of Southampton's $\mu$-VIS Centre ${ }^{1}$ using a Nikon Metrology HMX 225 CT scanner and an X-Tek Benchtop CT system. A molybdenum target with no filtering was used. SRCT scans were carried out at the European Synchrotron Radiation Facility (ESRF) in Grenoble, France, using beamline ID19, the sample-detector distance being set to $37 \mathrm{~mm}$ enabled near-field Fresnel diffraction (edge detection). The settings used for $\mu \mathrm{CT}$ and SRCT are shown in Table 1. These scans were reconstructed using a filtered back projection algorithm to form an 8-bit volume with voxel grey scale intensities ranging from 0-255.

The same specimens were scanned for all three imaging facilities and no penetrants or any other treatment was applied to the specimens. Whilst the full lengths of the cracks were not captured due to the limited field of view, the same regions of interest were obtained with each of the three imaging facilitates. This enabled the same cracks from the same specimens to be directly compared across the different imaging facilities.

\footnotetext{
${ }^{1}$ www.southampton.ac.uk/muvis, EPSRC Standard Research Grant EP/H01506X/1
} 


\subsection{Partial volume COD approximation technique}

As the size of the crack opening approaches the voxel resolution, a combination of background material and crack features are sampled and averaged within a voxel. Visually, the crack may appear faint, with a limited contrast against the surrounding material. This effect is shown in the lower resolution HMX scan in Figure 2(c) with a higher resolution SRCT scan of the same crack shown in Figure 2(b). Figure 3 illustrates this behaviour; a line plot across a $\sim 3$ micron crack exhibits a dip in grey scale value centred at $23 \mu \mathrm{m}$, which is indicative of the presence of a crack. The SRCT scan shows a sharp contrast between the crack and background material over a narrow band of voxels. In the example given in Figure 2(b) white fringes are present at the edge of the crack representing the edge detection regime. In this particular case, the fringe was more pronounced on the left hand side of the crack due to slight variations in path length through irregular material which has a control on the interference effect when reconstructed. With the lower resolution $\mu \mathrm{CT}$ scans, the crack intensity diminishes towards the background material mean grey scale, with the dip spread over a wider range of voxels, due to the crack partially filling the voxels.

By taking the grey scale intensity of a voxel partially containing a crack, the percentage of crack and material occupied within the voxel can be approximated as a linear relationship of a combination of grey scales representing the crack (i.e. air) and material. Provided isotropic voxels are used, this calculated percentage can be weighted to the corresponding length of the voxel. A linear relationship between the adjusted length of a voxel and the two grey scale intensity values consisting of the crack (air) and the material is represented by the relationship in Equation 1: 


$$
\delta_{i j}=\delta_{\text {res }}-\delta_{\text {res }}\left(\frac{g_{i j}-g_{\text {air }}}{g_{\text {mat }}-g_{\text {air }}}\right) \begin{cases}g_{i j}=g_{\text {air }} & \text { if } g_{i j} \leq g_{\text {air }} \\ g_{i j}=g_{\text {mat }} & \text { if } g_{i j} \geq g_{\text {mat }}\end{cases}
$$

this can be applied to approximate the adjusted length $\delta_{i j}$ at voxel ij where $\delta_{\text {res }}$ represents the length of one voxel resolution and $g_{i j}$ is the grey scale value at voxel $\mathrm{ij}$. To obtain the grey scale constant of the material, $\mathrm{g}_{\mathrm{mat}}$, a region representing material away from the crack is selected and the mean grey scale value is used; for the grey scale constant of the crack, $g_{a i r}$, the grey scale value of air at the centre of a large crack opening known not to be affected by any partial volume effects is used. When the sampled grey scale value is equal to or less than $g_{\text {air }}$ it is assumed that the whole voxel contains a crack and an adjusted width is applied equal to the width of the voxel resolution. Similarly if the voxel grey scale is equal to or greater than $\mathrm{g}_{\mathrm{mat}}$, it is assumed that no cracks are contained within that voxel and a zero length is applied. A grey scale value between the two limiting values will result in an adjusted length being applied based on the proportion of crack and material contained within the voxel.

After calculations of the adjusted length $\delta_{i j}$ at voxel $\mathrm{ij}$, these adjustments can be applied to approximate the mean $\mathrm{COD}, \bar{\delta}$, using Equation 2:

$$
\bar{\delta}=\frac{1}{N} \sum_{i=1}^{N} \sum_{j=1}^{M} \delta_{i j}
$$


In the case of a vertical crack such as that shown with a schematic in Figure 2(c), the crack opening on each horizontal row of voxels, $i$, is calculated by summing the adjusted lengths $\delta_{i j}$ at each voxel position along the row, $j$, in which $M$ represents the total number of voxels in the row. The CODs from each respective row are then summed together and divided by the number of rows $\mathrm{N}$ to give the mean estimated COD value $\bar{\delta}$ across all the rows.

To estimate the COD profile along the length of the crack in the $k$ direction (i.e. perpendicular to the plane of an image "slice" such as shown in figure 2), and to give a 3D representation, the equation was applied to one $2 \mathrm{D}$ slice at a time, thus obtaining a displacement for each slice along the length of the crack, equal to the voxel depth.

\subsection{Measurements of CODs by counting interconnected voxels}

To achieve COD measurements from $\mu \mathrm{CT}$ (HMX and Benchtop) image volumes, both matrix cracks and delaminations were segmented and binarised by thresholding. In a conventional approach, an ISO50\% threshold value is used consisting of a value exactly halfway between the mean air and material grey scale values [25]. The ISO50\% approach was used on SRCT scans using a threshold value halfway between the light and dark fringes of the crack.

In this study, the majority of crack openings in $\mu \mathrm{CT}$ scans were around or below the voxel resolution leading to partially filled cracks. Segmentation by the ISO50\% approach would exclude all partially filled cracks with voxels containing more than $50 \%$ material, therefore although the cracks could be detected, it will show no crack opening measurement using the ISO50\% method. 
To segment all detectable cracks in $\mu \mathrm{CT}$ scans, a threshold value was chosen to exclude the material. This was achieved by measuring the grey scale values within a region of composite material containing no cracks and taking the mean minus two standard deviations of the grey scale values, excluding $\sim 95 \%$ of the material. The crack was segmented by including values less than this calculated threshold value.

Voxels representing the segmented cracks were measured across the opening at all points across the crack and then averaged to calculate the mean crack opening; this is referred to in this study as the 'counting' method. In SRCT scans, due to COD measurement variations at different points across the crack, error bars representing the standard error of the mean COD are reported. This technique was applied to both the 2D and 3D COD measurements. It should be noted that the edge detection fringes and partial volume effects on the SRCT scans can also influence the accuracy of these measurement, up to the length of a single voxel; therefore the COD is taken as an average across multiple measurements.

\subsection{Comparisons between measured COD mean values}

In order to allow consistent comparisons, no modifications were made such as rotating, tilting, or repositioning the reconstructed volumes; which could affect the results by resampling the voxel intensities and positions. Direct comparisons were made from raw 8bit reconstructions on cracks obtained at identical positions between scans; with both $\mu \mathrm{CT}$ locations within one voxel of the equivalent position in the SRCT scan. This was achieved by measurements of the crack's position relative to fiducial features present in the scan such as small voids, inclusions, and other crack positions present in the volume.

By taking the mean COD obtained from SRCT measurements as the benchmark value, error values were calculated for the estimates obtained from the two $\mu \mathrm{CT}$ scans for each crack 
location. This allowed an assessment to be made of the partial volume COD approximation technique and also allowed comparisons with the method of counting interconnected voxels. Percentage errors were not calculated along 3D crack lengths. This is due to the different voxel resolutions used between the three CT scans resulting in the cross-sectional spacing no longer coinciding beyond the first slice, therefore preventing direct COD comparison using this method in the through-thickness direction along the length of the crack. Instead the general trends between COD and crack length are presented and compared.

\section{Results and Discussion}

\subsection{Partial volume COD assessment}

Figure 4(a) shows an example of a delamination as indicated by the box: close up SRCT and

$\mu \mathrm{CT}(\mathrm{HMX}$ System) images of this delamination are shown in (b-i) and (c-i) respectively and has been binarised by thresholding in (b-ii) and (c-ii). It is clear that due to the crack partially filling neighbouring voxels, segmentation of detectable cracks (c-ii) has captured a region larger than the true opening of the crack shown in (c-i), and when the opening is measured as indicated by the arrow, it is overestimated.

Measurement of the CODs of the delamination in Figure 4 is shown in a plot in Figure 5 as a function of voxel position along the crack width. The COD measurement from $\mu C T$ scanning is shown to overestimate the crack opening by approximately three times. Applying the partial volume correction to the HMX scan, the estimated COD is comparable to SRCT measurements. Interestingly, some local variation in the SRCT COD measurements are observed depending on the position of the measurement due to the nature of the crack 
morphology; these variations have been smoothed by the partial volume correction estimation and fall within the peak to peak regions in the SRCT measurements. When the average CODs are considered consisting of $4.9 \mu \mathrm{m}, 4.8 \mu \mathrm{m}$ and $17.4 \mu \mathrm{m}$ for the SRCT, $\mu \mathrm{CT}$ partial volume correction and $\mu \mathrm{CT}$ counting measurements respectively, it is clear that simple linear partial volume considerations provide a good estimation of crack opening from the lower resolution the $\mu \mathrm{CT}$ scan.

\subsection{Average COD comparisons on single slice cracks}

The partial volume correction algorithm was applied to $\mu \mathrm{CT}$ scans to estimate the average CODs on several 2D matrix cracks and delaminations. These were compared to counting COD measurements and SRCT measurements. The results of crack opening measurements are shown in Figure 6(a) for particle toughened and Figure 6(b) untoughened resin systems. Standard error bars indicated on SRCT COD measurements were larger in cases in which the cracks were more open, suggesting larger variations in crack openings at these levels. $R$ squared values on partial volume correction estimates were 0.68 and 0.87 for the toughened and non-toughened systems respectively. This scatter is believed to be principally caused by the ligamented and non-continuous delaminations; characteristic of particle toughening that could be detected in the SRCT scan but not in the $\mu$ CT scans. This led to bridged sites that were included in the partial volume correction leading to underestimations of the mean crack opening. Additional sources of scatter in the data include noise, beam hardening, ring artefacts, non-linearity in X-ray detector response and the simplicity of the algorithm which neglects the three-dimensionality of the partial volume effect. 
It should be noted that due to the use of different voxel resolutions across the three imaging facilities, there are approximately three to four SRCT cross-sectional slices to one $\mu \mathrm{CT}$ slice. Despite this issue, this has shown not to have significantly affected the partial volume correction results. It is probable that as the crack path moves through the $\mu \mathrm{CT}$ slice, the crack opening does not vary significantly at these voxel sizes, therefore voxels contain similar proportions of crack and material between $\mu \mathrm{CT}$ and SRCT scans.

In comparison, counting COD measurements on $\mu \mathrm{CT}$ scans overestimated CODs by up to five times. There is no correlation between the measurements obtained through counting connected voxel measurements in HMX and Benchtop scans and the measured SRCT scan. This shows the unreliability in using the counting technique in detectable but partially filled cracks to gauge crack openings. The use of partial volume correction is shown to reduce these significant overestimations.

Percentage errors comparing estimated $\mu \mathrm{CT}$ COD estimates to SRCT measurements are shown in Figure 7 and demonstrate good approximations within $20 \%$ error. Discontinuities in the crack generally led to underestimations either when the COD was below approximately $4 \mu \mathrm{m}$, or in the particle toughened system where delamination crack ligaments were present. The discontinuous behaviour of the cracks led to lower proportion of crack partially filling a voxel and hence an underestimation of the COD.

For counting interconnected voxel measurements in $\mu \mathrm{CT}$ scans, the percentage errors increase with smaller crack openings up to $500 \%$ and all measurements were overestimated. This demonstrates the unreliability of this method for measuring crack openings where the voxel resolution is within two to three voxels of the feature size, and the use of partial volume correction is shown it provide better accuracy. 


\subsection{D COD Assessments and Comparisons}

Multiple COD measurements were taken along the length of the crack at arbitrary starting and ending positions that fell within the field of view of the CT scans. These are shown in Figure $8(a-d)$ in which partial volume COD corrections are applied to HMX and Benchtop $\mu C T$ scans, and the resulting estimates are compared to those obtained from SRCT data.

Different crack types were measured consisting of delaminations as shown in Figure $8(a \& b)$ and matrix cracks as shown in Figure $8(c \& d)$. The COD measurements were made on the two materials; particle toughened as shown in Figure $8(\mathrm{a} \& \mathrm{c})$ and untoughened as shown in Figure $8(b \& d)$.

All measurements show similarly decreasing crack openings towards the crack tip. Comparisons between estimates from $\mu \mathrm{CT}$ observations to SRCT measurement show good COD correlations along the crack towards the crack tip. Divergence from the SRCT measurements started to occur when the COD was less than approximately $4 \mu \mathrm{m}$ leading to underestimations of the COD. This again was attributed to cracks becoming discontinuous below this threshold. The delamination COD estimations for toughened material, as shown in Figure $8(\mathrm{c})$, had the greatest scatter for both Benchtop and $\mathrm{HMX} \mu \mathrm{CT}$; this was attributed to the ligamented, non-continuous delamination associated with particle toughening.

The presence of ring artefacts created noticeable spiked peaks, particularly in the Benchtop $\mu \mathrm{CT}$ results in Figure $8(\mathrm{a} \& \mathrm{c})$. In this case the cracks were in a region of ring artefacts that affected the local grey scale intensity [13] which led to erroneous COD measurements. Care therefore needs to be taken to check the presence of neighbouring artefacts when taking partial volume correction estimations that are dependent on grey scale intensity values. 
Again, as discussed earlier, when the partial volume correction method is compared to the method of counting interconnected voxels representing the COD, improvements to the accuracy are achieved.

\section{Conclusions}

Crack opening displacement measurements were estimated on $\mu$ CT scans of impacted CFRP material by applying a partial volume correction based on grey scale intensities and weighting this to the voxel length. In 2D studies, partial volume estimates of CODs correlated well with measurements taken from higher resolution SRCT scans. This method is significantly more accurate (within $20 \%$ of SRCT measurements) than the more straightforward approach of simply counting interconnected voxels from detectable but partially filled cracks which overestimated CODs by up to $500 \%$. Underestimations occurred when the cracks became discontinuous, this was typically when the crack openings were less than approximately $4 \mu \mathrm{m}$, and in the toughened system where particles created crack ligaments in the delamination. Additional contributions to errors also include noise, nonlinearity in X-ray detector response, CT artefacts (ring artefacts and beam hardening), and the three-dimensional nature of the partial volume averaging process. The partial volume correction technique has been demonstrated as a means to obtain 3D crack opening profiles along the length of cracks. Good correlations were obtained with SRCT data in estimating crack openings along the length of the crack. Issues with the measured crack opening being distorted by ring artefacts led to erroneous results indicated by large peaks in the measurements. Overall the use of partial volume corrections has been shown to be a viable 
method to obtain quantitative estimates of crack opening displacements in composite materials using micro-focus computed tomography.

\section{Acknowledgements}

The author wishes to thank the European Synchrotron Radiation Facility in France for use of beamline ID19 and the $\mu$-Vis centre at the University of Southampton in the UK which enabled these experiments to be performed. Additional thanks are due to the Engineering and Physical Sciences Research Council (EPSRC) and Cytec Engineered Materials Ltd for financial support. The authors thank Dr. Kingsley Ho and Mr. Sam Hill at Cytec for their technical support for the project.

\section{References}

[1] Sugimoto S, Aoki T, Iwahori Y, Ishikawa T. Nondestructive evaluation of composites using Micro-Focused X-Ray CT Scanner. Aip Conf Proc. 2005;760:1081-1086.

[2] Schilling PJ, Karedla BPR, Tatiparthi AK, Verges MA, Herrington PD. X-ray computed microtomography of internal damage in fiber reinforced polymer matrix composites. Compos Sci Technol. 2005;65(14):2071-2078. [3] Tan KT, Watanabe N, Iwahori Y. X-ray radiography and micro-computed tomography examination of damage characteristics in stitched composites subjected to impact loading. Compos Part B-Eng. 2011;42(4):874-884. [4] Girshovich S, Gottesman T, Rosenthal H, Drukker E, Steinberg Y. Impact Damage Assessment of Composites. Am Soc Test Mater. 1992;1128:183-199. [5] Bathias C, Cagnasso A. Application of X-Ray Tomography to the Nondestructive Testing of High-Performance Polymer Composites. Am Soc Test Mater. 1992;1128:35-54.

[6] Pandita SD, Falconet D, Verpoest I. Impact properties of weft knitted fabric reinforced composites. Compos Sci Technol. 2002;62(7-8):1113-1123.

[7] Enfedaque A, Molina-Aldareguia JM, Galvez F, Gonzalez C, Llorca J. Effect of Glass Fiber Hybridization on the Behavior Under Impact of Woven Carbon Fiber/Epoxy Laminates. J Compos Mater. 2010;44(25):3051-3068.

[8] Arabi H, Asl ARK, Aghamiri SM. The effect of focal spot size on the spatial resolution of variable resolution X-ray CT scanner. Iran J Radiat Res. 2010;8(1):37-43. 
[9] Wright P, Moffat A, Sinclair I, Spearing SM. High resolution tomographic imaging and modelling of notch tip damage in a laminated composite. Compos Sci Technol. 2010;70(10):1444-1452.

[10] Withers PJ, Bennett J, Hung YC, Preuss M. Crack opening displacements during fatigue crack growth in $\mathrm{Ti}-\mathrm{SiC}$ fibre metal matrix composites by X-ray tomography. Mater Sci Tech-Lond. 2006;22(9):1052-1058.

[11] Hoffman EJ, Huang SC, Phelps ME. Quantitation in Positron Emission Computed-Tomography .1. Effect of Object Size. J Comput Assist Tomo. 1979;3(3):299-308.

[12] Plewes DB, Dean PB. The Influence of Partial Volume Averaging on Sphere Detectability in Computed-Tomography. Phys Med Biol. 1981;26(5):913-919. [13] Barrett JF, Keat N. Artifacts in CT: Recognition and Avoidance. Radiographics. 2004;24(6):1679-1691.

[14] Gates M, Lambros J, Heath MT. Towards High Performance Digital Volume Correlation. Exp Mech. 2011;51(4):491-507.

[15] Buffiere JY, Maire E, Adrien J, Masse JP, Boller E. In Situ Experiments with X ray Tomography: an Attractive Tool for Experimental Mechanics. Exp Mech. 2010;50(3):289-305.

[16] Soret M, Bacharach SL, Buvat I. Partial-volume effect in PET tumor imaging. J Nucl Med. 2007;48(6):932-945.

[17] Oliveira CAP, Meurer MI, Pascoalato C, Silva SRC. Cone-beam computed tomography analysis of the apical third of curved roots after mechanical preparation with different automated systems. Brazilian Dental Journal. 2009;20:376-381.

[18] Pham DL, Xu CY, Prince JL. Current methods in medical image segmentation. Annu Rev Biomed Eng. 2000;2:315-+.

[19] Heckel F, Dicken V, Bostel T, Fabel M, Kiessling A, Peitgen HO. Partial volume correction for volume estimation of liver metastases and lymph nodes in CT scans using spatial subdivision. P Soc Photo-Opt Ins. 2010;7623. [20] Conradi SH, Lutey BA, Atkinson JJ, Wang W, Senior RM, Gierada DS. Measuring Small Airways in Transverse CT Images: Correction for Partial Volume Averaging and Airway Tilt. Acad Radiol. 2010;17(12):1525-1534.

[21] Ignatiev KI, Davis GR, Elliott JC, Stock SR. MicroCT (microtomography) quantification of microstructure related to macroscopic behaviour - Part 1 Fatigue crack closure measured in situ in AA 2090 compact tension samples. Mater Sci Tech-Lond. 2006;22(9):1025-1037.

[22] Guvenilir A, Breunig TM, Kinney JH, Stock SR. New direct observations of crack closure processes in Al-Li 2090 T8E41. Philos T Roy Soc A.

1999;357(1761):2755-2775.

[23] Guvenilir A, Breunig TM, Kinney JH, Stock SR. Direct observation of crack opening as a function of applied load in the interior of a notched tensile sample of Al-Li 2090. Acta Mater. 1997;45(5):1977-1987.

[24] Sanchu-Saez S, Barbero E, Zaera R, Navarro C. Compression after impact of thin composite laminates. Compos Sci Technol. 2005;65(13):1911-1919. 
[25] Kiekens K, Welkenhuyzen F, Tan Y, Bleys P, Voet A, Kruth JP, et al. A test object with parallel grooves for calibration and accuracy assessment of industrial computed tomography (CT) metrology. Meas Sci Technol. 2011;22(11).

a)

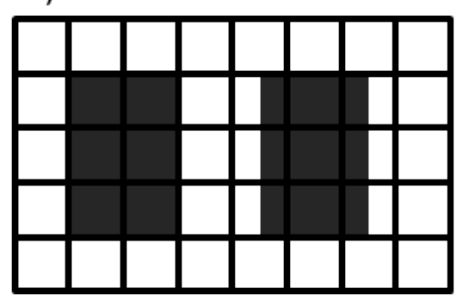

b)

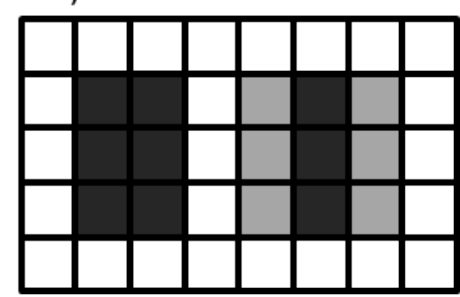

Figure 1 - Schematic showing (a) positions of an actual crack relative to the image grid, (b) corresponding rendered image. The crack boundaries that partially spill over and fill neighbouring voxels are rendered with less intensity at the edges and result in an inaccurate width estimate, this leads to consistent overestimates when measured by counting interconnected voxels. 

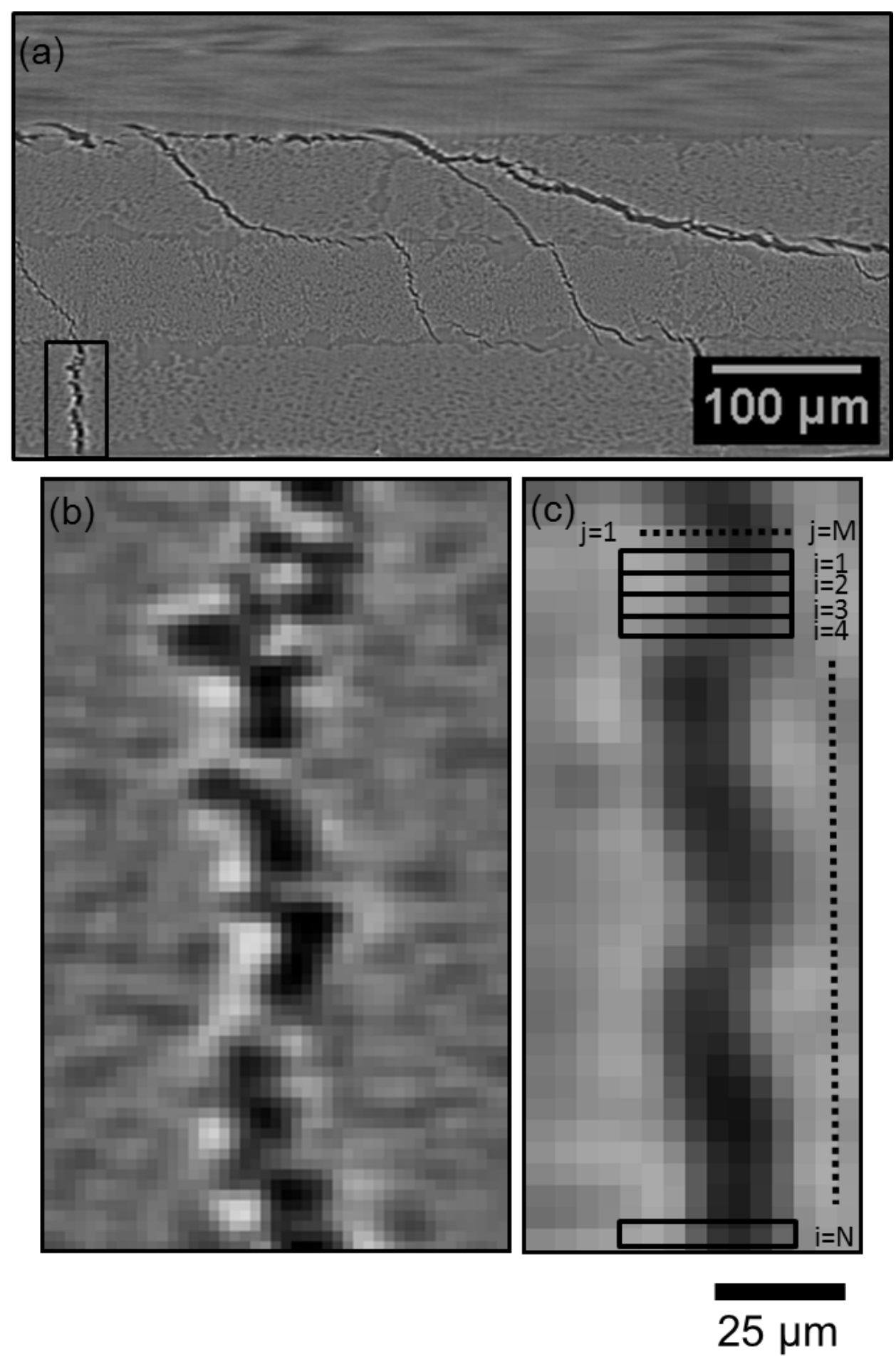

Figure 2 - (a) SRCT cross-section showing matrix cracks and delaminations, the box highlights highlight a close up of this region in (b) and for an HMX scan of the same crack in (c). A schematic in (c) also shows the sampled rows and columns used to estimate the COD in equation 1. 


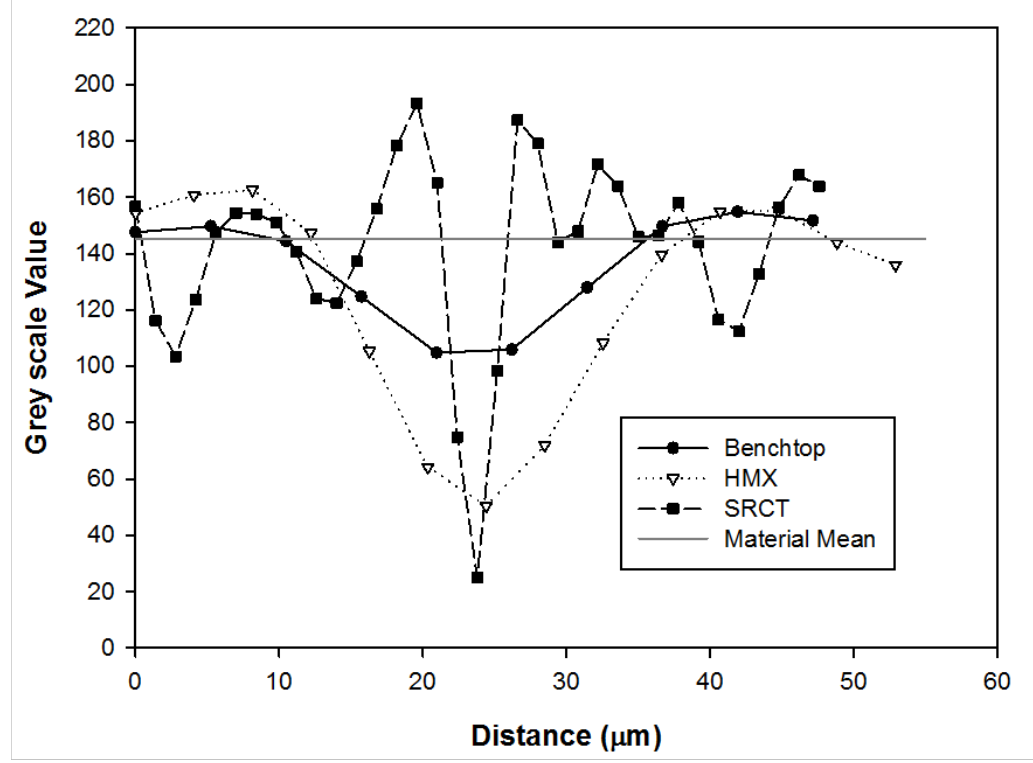

Figure 3 - Grey scale intensity line plot across the same crack obtained from three different CT scans.
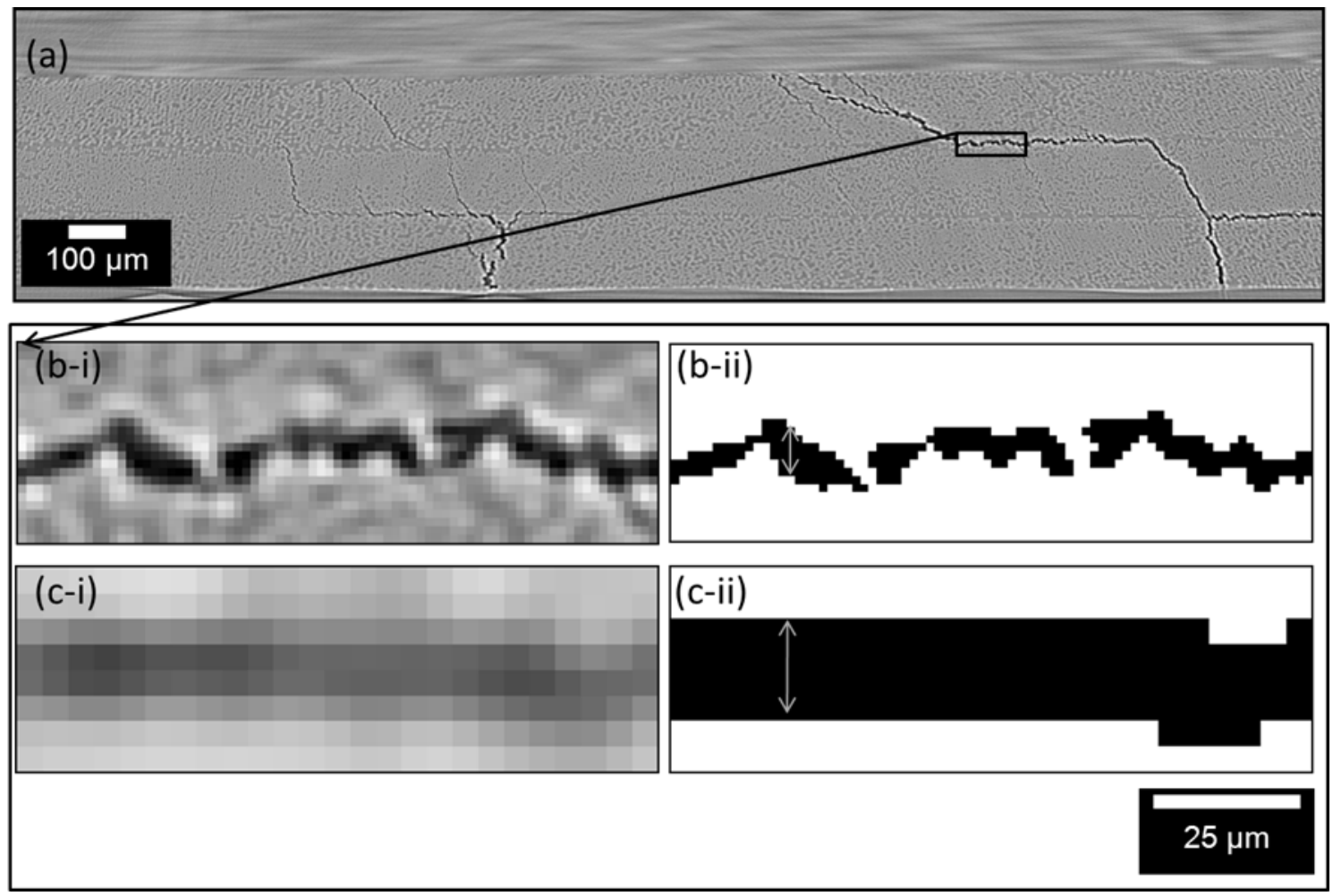

Figure 4 - An example of counting interconnected voxels to measure the COD. A cross-section of this damage is shown in the SRCT scan in (a), the box indicates a close up of this SRCT region in (b-i) and for an HMX scan in (c-i). Binarised images from these cracks are shown in (b-ii) and (c-ii), the arrow indicating the method of counting interconnected voxels representing the COD. 


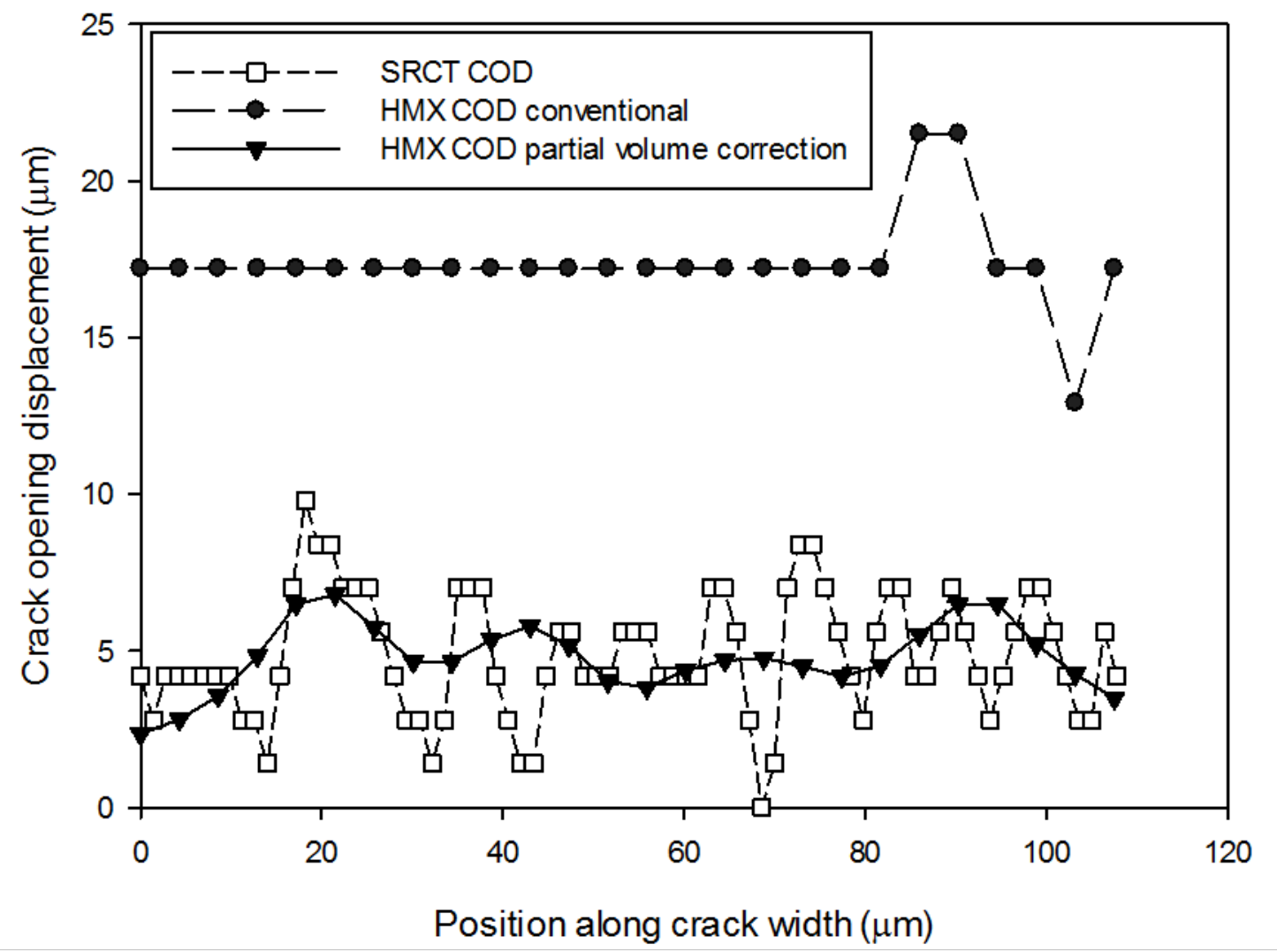

Figure 5-COD measurements of the delamination shown in Figure 5. SRCT and HMX COD were measured at each voxel along the width of the crack. A partial volume correction shows an improvement to the HMX COD measurement.

(a) Particle toughened

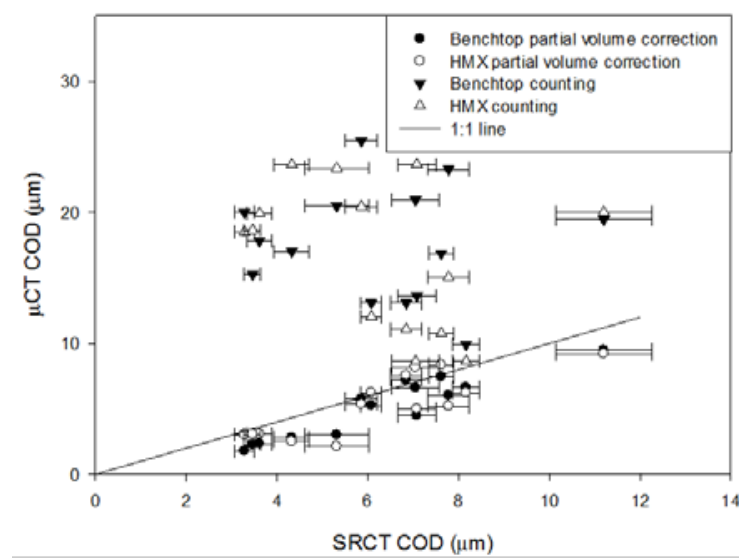

(b)

Untoughened

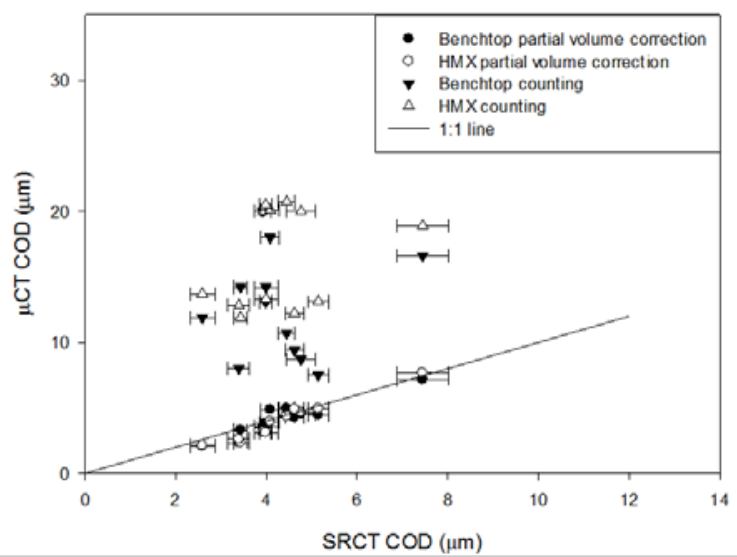

Figure 6 - Graph showing partial volume corrected and counting COD measurements obtained from $\mu \mathrm{CT}$ scans directly compared to SRCT COD measurements for (a) toughened and (b) non-toughened specimens. 
(a) Particle toughened

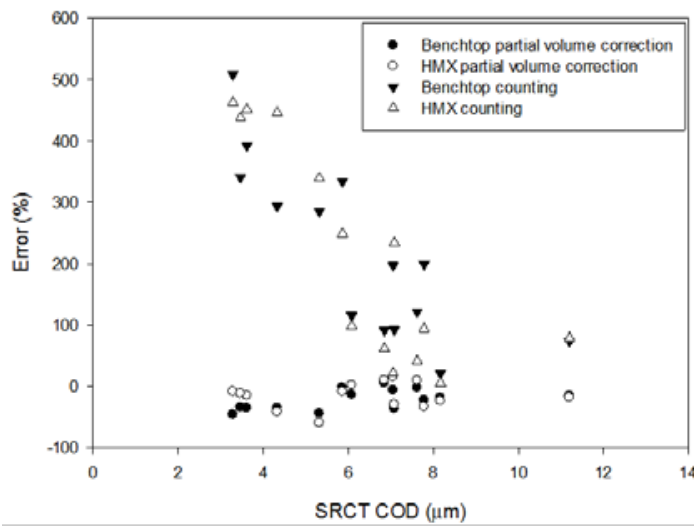

(b)

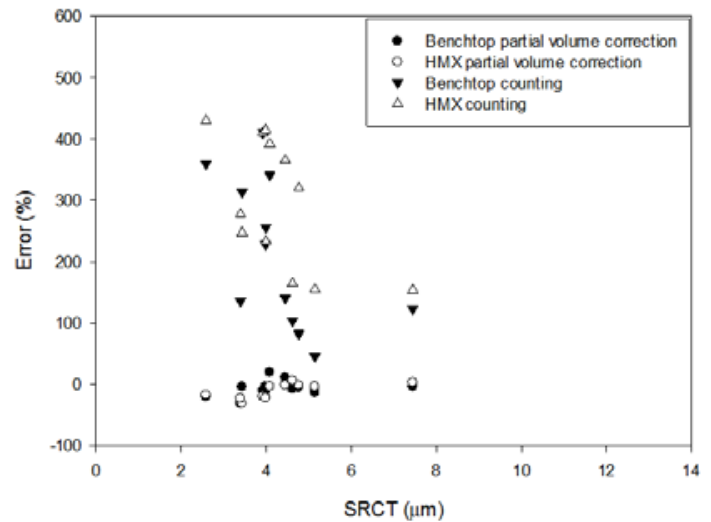

Figure $7-C O D$ percentage errors for counting and partial volume corrected measurements compared to SRCT measurements for (a) particle toughened and (b) untoughened material

(a) Particle toughened delamination

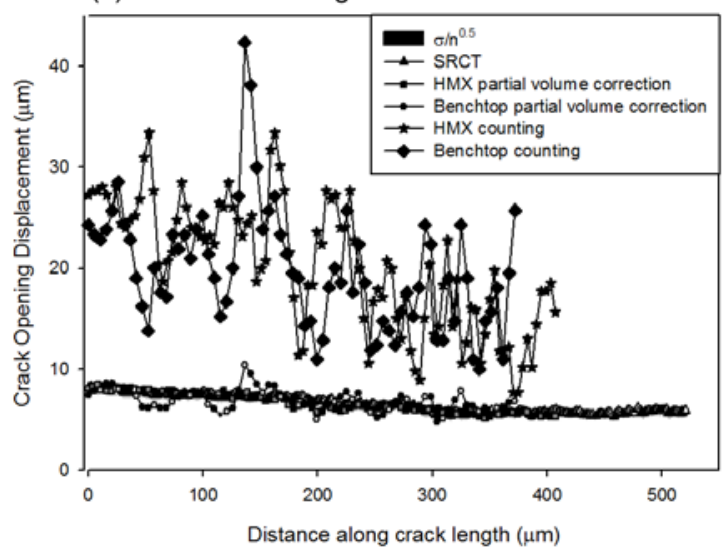

(c) Particle toughened matrix crack

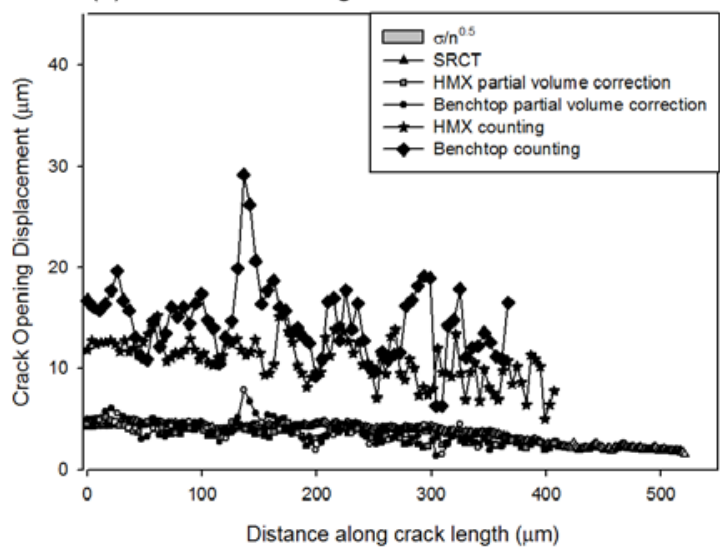

(b) Untoughened delamination

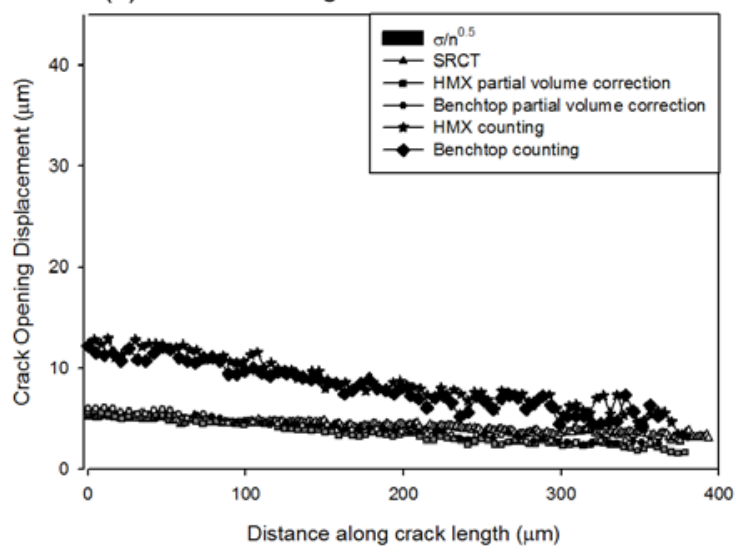

(d) Untoughened matrix crack

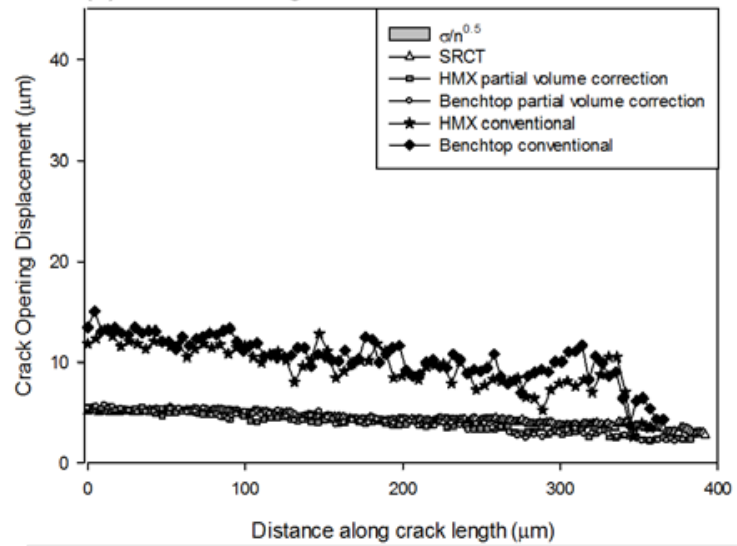

Figure 8- COD measurements along the length of a crack comparing counting and partial volume correction techniques applied to HMX and Benchtop CT cracks to SRCT measurements. 


\begin{tabular}{llll}
\hline & Benchtop & HMX & SRCT \\
\hline Energy $(\mathrm{kV})$ & $75($ peak) & 65 (peak) & 19 (monochromatic) \\
\hline Current $(\mu \mathrm{A})$ & 80 & 70 & - \\
\hline $\begin{array}{l}\text { Reconstruction size } \\
(\mathrm{px})\end{array}$ & $1000 \times 1000$ & $2000 \times 2000$ & $2000 \times 2000$ \\
\hline Voxel resolution $\left(\boldsymbol{\mu \mathrm { m } ^ { 3 } )}\right)$ & 5.24 & 4.3 & 1.4 \\
\hline $\begin{array}{l}\text { Number of } \\
\text { radiographs }\end{array}$ & $2000\left(360^{\circ}\right)$ & $2000\left(360^{\circ}\right)$ & $1500\left(180^{\circ}\right)$ \\
\hline Exposure time $(\mathrm{ms})$ & 2,000 & 2,000 & 100 \\
\hline Scan time (mins) & 150 & 150 & 5 \\
\hline
\end{tabular}

